\title{
La corporeidad como estrategia para el desarrollo de habilidades y preservación de la salud en escolares en la nueva normalidad
}

\author{
Corporeity as a strategy for the development of abilities \\ and preservation of health in students in the new normality
}

Ricardo Juárez lozano • Luis Manuel lara Rodríguez • Gabriel Medrano Donlucas

Ricardo Juárez Lozano. Universidad Autónoma de Ciudad Juárez, Chihuahua, México. Profesor-investigador de tiempo completo con perfil Prodep desde el 2005. Es doctor en Educación Física y Deporte por la Universidad de Lleida, España; maestro en Administración y licenciado en Educación Física por la Universidad Autónoma de Chihuahua. Autor de diversas publicaciones en revistas indizadas, libros, capítulos de libros y conferencias en congresos nacionales e internacionales. Reconocimiento a las mejores prácticas universitarias (CEMEFI, 2017). Fundador del programa sociocultural MARACAS. Miembro del Cuerpo Académico 112: Cultura Física, Educación y Sociedad. Correo electrónico: rjuarez@uaci.mx. ORCID: https:// orcid.org/0000-0002-1468-5384.

Luis Manuel Lara Rodríguez. Universidad Autónoma de Ciudad Juárez, Chihuahua, México. Es doctor en Ciencias Sociales, sociólogo. Responsable de proyectos federales en intervención social con eje deportivo (2013-2017). Docente en maestrías en Ciencias Sociales, Estudios Interdisciplinarios de Género, Investigación Educativa Aplicada, y de pregrado en licenciaturas de Sociología y de Entrenamiento Deportivo. Responsable del Cuerpo Académico Cultura Física, Educación y Sociedad. Ponente en eventos nacionales e internacionales (Costa Rica, Uruguay, Argentina, Chile, Colombia, Estados Unidos y España). Publicaciones recientes: libros Cultura física
Resumen

A partir de la teoría histórico-cultural de la actividad, el desarrollo de los sujetos es potenciado por la enseñanza y en general por los sistemas de actividad y comunicación en que ellos se insertan. En función de ello, el programa sociocultural Motivando la Adecuada Recreación Activa y la Correcta Alimentación Saludable (MARACAS) tiene como objetivo ser un programa multidisciplinar de actividad física y deporte basado en el desarrollo de habilidades para la vida desde una perspectiva de inteligencias múltiples. El presente artículo pretende informar los resultados principales obtenidos en la dimensión psicológica del programa en una experiencia veraniega del 2019. Para obtener los resultados se recabaron datos a lo largo del programa y los mismos fueron procesados cuantitativamente en el Paquete Estadístico para las Ciencias Sociales (SPSS), versión 25.0. Los resultados apuntan hacia una diferenciación en cuanto a género a la hora de optar por insertarse en el programa, así como a una fuerte influencia de la cultura familiar en la decisión de practicar deportes. Las habilidades más desarrolladas en los participantes fueron las físico-motoras y presentan un alto desarrollo de sus habilidades kinestésico-corporales. Se discuten las implicaciones de tales resultados como una medida alternativa de favorecer la corporeidad en el educando durante periodos vacacionales. Ante la nueva normalidad, la educación física en el nivel de educación básica se vuelve una asignatura indispensable para la preservación de la salud y la adquisición de estilos de vida saludables.

Palabras clave: Cuerpo, educación física, salud, estudiantes, desarrollo de habilidades. 
e intervención comunitaria (UACJ) y Deporte y género (UACJ-UAMEX). Correo electrónico: luis.lara@uacj.mx. ORCID: https://orcid. org/0000-0003-3112-5140.

Gabriel Medrano Donlucas. Universidad Autónoma de Ciudad Juárez, Chihuahua, México. Es profesor de tiempo completo en la UACJ. Licenciado en Nutrición y maestro en Docencia Biomédica. Diplomado Educador en Diabetes y en Obesidad y Nutrición, ambos organizados por la Asociación Mexicana de Diabetes. Actualmente ocupa la jefatura de la Unidad Administrativa del ICB-UACJ. Coordinador del Programa en Nutrición en la UACJ en el periodo 2006-2018. Colaborador en el proyecto nacional "Composición corporal por el modelo molecular de cuatro compartimientos en adultos mayores con obesidad de la zona norte de México: datos para el desarrollo de ecuaciones precisas, exactas y prácticas". Correo electrónico: gmedrano@uacj.mx. ORCID: https://orcid.org/0000-0001-6349-2338.

\begin{abstract}
From the cultural historical activity theory, the development of the subjects is enhanced by teaching and in general by the systems of activity and communication in which they are inserted. Based on this, the project Motivando la Adecuada Recreación Activa y la Correcta Alimentación Saludable (Maracas, "Motivating Adequate Active Recreation and Correct Healthy Eating"), aims to be a program of physical activity and sport based on the development of life skills from a perspective of multiple intelligences. This article aims to inform the main results obtained in the psychological dimension of the project. To obtain the results, data were collected throughout the entire project and they were processed quantitatively in the Statistical Package for Social Sciences (SPSS), v. 25.0. The results point towards a differentiation in terms of gender when choosing to enter the program, as well as a strong influence of family culture in the decision to practice sports. The most developed skills in the participants were motor physics and they present a high development of their kinesthetic-bodily skills. The implications of such results are discussed as an alternative measure of promoting corporeity in the student during their vacation periods. Faced with the new normality, Physical Education at the basic education level becomes an indispensable subject for the preservation of health and the acquisition of healthy lifestyles.
\end{abstract}

Keywords: Body, physical education, students, skill development.

\section{Contexto}

El impacto de la inactividad física en escolares se observa desde dos vertientes: la primera desde el punto de vista de la salud, y la segunda a partir de la violencia y la delincuencia. En la primera, la inactividad física se ha convertido en un problema de salud pública en México. Datos de la UNESCO (2018) señalan que más del 80\% de los niños de 10 a 14 años y cerca del 40\% de los adolescentes de 15 a 19 años son inactivos, y las evidencias indican que este factor de riesgo continúa en aumento. Asimismo, la Encuesta Nacional de Salud y Nutrición 2018 muestra la prevalencia de sobrepeso y obesidad en niños de 5 a 11 años con 35.6\%, jóvenes de 12-19 años con $38.4 \%$, y la población de 20 años y más con 73\% (ENSANut, 2018).

Tan solo en el nivel básico en México (preescolar, primaria y secundaria) ciclo escolar 2019-2020 se inscribieron 25’780,693 alumnos (3-14 años) dentro del Sistema Educativo Escolarizado, de los cuales 734,845 corresponden al estado de Chihuahua (SEP, 2019).

Ciertamente desde la SEP se llevan a cabo planes y programas de estudio sobre la asignatura de Educación Física, el más reciente denominado "Aprendizajes claves para la formación integral”, en donde se manifiesta que la educación física es una forma de intervención pedagógica que contribuye al desarrollo integral de niñas, niños y 
adolescentes mediante el desarrollo de la motricidad e integración de la corporeidad. ${ }^{1}$ Es decir, constituye en los centros escolares de educación básica (preescolar, primaria y secundaria) el espacio curricular que moviliza al cuerpo (corporeidad y motricidad) y fomenta el gusto por la actividad física. La finalidad formativa de la educación física en el contexto escolar es la edificación de la competencia motriz por medio de la corporeidad. Asimismo, en los rasgos del perfil de egreso de la educación básica se afirma la atención al cuerpo y la salud de los estudiantes.

La corporeidad se manifiesta por medio de gestos, posturas, expresiones corporales y sus diferentes acciones motrices, relacionadas con las emociones y sentimientos que cada alumno experimenta. Es una construcción permanente que las personas hacen de sí; una unidad que fusiona la parte física y funcional del cuerpo con el aspecto cognitivo, social, personal y cultural. Es decir, la expresión y manifestación de las habilidades para la vida (SEP, 2017). Es aquí donde recae nuestro punto de interés, ya que favorecer este componente equivale a amalgamar las experiencias, así como asociar el conocimiento de sí con saberes, habilidades, actitudes y valores para la vida. Para tal efecto, el campamento de verano educativo de la UACJ contribuye a la integración de la corporeidad, ya que establece conexiones con los procesos de decisión, anticipación y comprensión en cada uno de sus escenarios de aprendizaje; es decir, el cuerpo mismo visto desde una perspectiva integradora y no únicamente físico-corporal, de rendimiento o de eficiencia, sino el cuerpo en constante movimiento; correr, saltar, trepar, lanzar, atrapar, botar, caminar, trotar, driblar, entre otras, son acciones motrices que involucran habilidades para la vida (cognitivas, sociales, personales y físico-motrices).

La segunda vertiente se ha convertido en un problema social sin precedentes en los últimos 20 años. Las tres principales causas de muerte en México registradas entre enero y agosto del 2020 son enfermedades del corazón (141,873; 20\%), Covid-19 $(108,658 ; 15 \%)$ y diabetes mellitus (99 733, 14.6\%); mientras que los homicidios se colocan en la octava causa de muerte a nivel nacional, con 22,798 (INEGI, 2020).

Sin embargo, los datos muestran que entre los jóvenes de 11 a 17 años la principal causa de muerte es el homicidio, con 12\%, mientras que de 18 a 25 años $32.7 \%$ por la misma causa. Es decir, los están y se están matando entre los jóvenes en su mayoría ligados al crimen organizado. Ante esta grave problemática, ¿cuál es el papel de la educación básica con respecto a estos jóvenes?, ¿qué estrategias se están implementando y cuáles son sus mecanismos de evaluación?

La teoría de la actividad histórico-cultural plantea que el aprendizaje precede al desarrollo, para lo cual se vuelve indispensable la perspectiva de Vigotsky y sus

1 Corporeidad: construcción permanente que las personas hacen de sí; una unidad que fusiona la parte física y funcional del cuerpo con el aspecto cognitivo, social, personal y cultural. Es decir, la expresión y manifestación de las habilidades para la vida. 
conceptos de zona de desarrollo próximo (ZDP) y situación social del desarrollo (SSD) (Arias, 1994).

Entender esto es esencial para vislumbrar cómo se articula la relación dialéctica entre lo externo y lo interno en el desarrollo humano. La edad escolar, por ejemplo, se caracteriza por sistemas de comunicación y actividad en los que la educación formal tiene un papel rector y la comunicación educativa del maestro, así como la resolución de tareas formales de estudios, permiten el paso a las nuevas formaciones psicológicas que determinan el desarrollo del niño. En el caso del desarrollo de habilidades, la ecuación, según este enfoque, sería similar. Las habilidades no se desarrollan por maduración ni por la mera incorporación de hábitos, sino en la adecuada relación entre procesos internos (ejemplo: motivación por la actividad física), y por sistemas de actividad y comunicación orientados de manera planificada a su desarrollo.

El cuerpo es un instrumento maravilloso al servicio del lenguaje y la comunicación, el cual se expresa a través del movimiento y, en nuestro caso, a través de la corporeidad. Según la teoría de Breton (2007), el cuerpo es un instrumento de comunicación social, en el que se utilizan todos los sentidos para la comprensión del mundo y del contexto.

\section{Problema de estudio}

Obesidad y violencia son dos grandes problemas sociales que aquejan a los mexicanos; especialmente en el estado de Chihuahua, la obesidad infantil ocupa los primeros lugares en incidencia. Los malos hábitos alimenticios y la falta de actividad física han ocasionado que los niños sean víctimas potenciales de esta pandemia. Es un problema multifactorial, pues depende de los estilos de vida: alimentación, sedentarismo, entorno social, educación formal y no formal, familia, entre otros factores asociados (ENSANUT, 2018).

Por otro lado, los índices de homicidios asociados al narcotráfico suelen atraer a sus redes a niños y jóvenes a quienes el sistema educativo no ha sabido retener y/o brindar oportunidades para desarrollar sus talentos y habilidades diversas.

Ante este panorama, podemos preguntarnos lo siguiente: ¿Los campamentos de verano educativos de la UACJ estimulan las inteligencias múltiples y habilidades para la vida de los participantes? ¿Es posible incorporar la corporeidad como una estrategia de preservación de la salud, en un contexto educativo no formal como el campamento de verano UACJ?

\section{Objetivos}

- Analizar la frecuencia de las inteligencias múltiples y habilidades para la vida en los participantes del campamento de verano educativo UACJ.

- Analizar la corporeidad como una estrategia de preservación de la salud en escolares en un contexto educativo no formal. 


\section{Metodología}

La intención fue analizar los dominios de los participantes a través de los escenarios de aprendizaje, ${ }^{2}$ con actividades atractivas y enfocadas a resaltar aquellos aspectos que más destacasen en los implicados. Por otro lado, fue necesario un sistema de análisis que diera significado a los avances observados en los sujetos a través de las actividades deportivas. Para ello, el presente diseño se adaptó a un estudio etnográfico educativo. Se adecuaron registros de observación para evaluar y estudiar las capacidades de los participantes desde ocho maneras de expresar el potencial, según la teoría de las inteligencias múltiples (Gardner, 1987). Con base en lo anterior, y siguiendo criterios de Álvarez (2007), se utilizó un sistema constituido por ocho categorías, poniendo principal atención en aquellas características que potencian cada uno de los participantes.

La observación pasó por diferentes estadios. El primero lo constituye la elección del entorno donde se realizará (Álvarez, 2007). El estudio se desarrolló en las instalaciones deportivas de la $\mathrm{UACJ}^{3}$ en el periodo vacacional de verano del 2019. Segundo, se estableció un equipo de observación con alumnos de prácticas profesionales que habrían cursado previamente un curso de formación y capacitación, ${ }^{4}$ además se les facilitaron herramientas precisas para el análisis de situaciones específicas. Tercero, el equipo tomó parte estratégica en las actividades realizadas por los participantes, favoreciendo la interacción social y propiciando escenarios para expresar sus preferencias, gustos, habilidades y capacidades mediante las actividades veraniegas. Cuarto, se diseñó un registro que nos permitió identificar y valorar los momentos en que se presentaban las habilidades de los participantes, así como el progreso de las inteligencias múltiples observadas. Los datos que se presentarán a continuación fueron recabados por practicantes de las licenciaturas en Educación, Psicología y Entrenamiento Deportivo.

Se recolectó la información a partir de la aplicación del Test de Inteligencias Múltiples de Gardner, así como de la revisión de los formatos de inscripción y llenado diario de formatos de observación sobre habilidades para la vida. Utilizamos una escala en la cual el observador escoge la categoría o clasificación que mejor represente o describa el objeto de la evaluación, cuya estimación fue la siguiente: nunca 0 , rara vez 1, de vez en cuando 2, con frecuencia 3, siempre 4 (McKernan, 2008).

Los participantes fueron observados en todo momento por periodos de $50 \mathrm{mi}$ nutos en cuatro clases por día durante el mes de julio (ver tabla 1).

2 Son espacios de aprendizaje controlados que se generan a través de las actividades deportivas en que participan los estudiantes en el programa sociocultural MARACAs, lo cual es aplicable en distintos contextos como educación formal y no formal.

3 Complejo Deportivo Universitario de la Universidad Autónoma de Ciudad Juárez.

4 Estudiantes de Educación, Piscología y Entrenamiento Deportivo de la UACJ, participan en un curso para tutores previo a la intervención. 
Tabla 1. Tiempo estimado de observación por grupo.

\begin{tabular}{ccccc} 
Por clase & Por día & Por semana & Por mes & Tiempo total \\
\hline 50 minutos & 200 minutos & 1,000 minutos & 4,000 minutos & 4,000 minutos \\
\hline \multicolumn{5}{c}{ Fuente: Elaboración propia. }
\end{tabular}

Una vez recabados los datos, fueron capturados en Excel y trasladados para su posterior procesamiento en el Paquete Estadístico para las Ciencias Sociales (SPSS), versión 25.0. Para el análisis se utilizaron solo aquellos registros que no tuvieron ningún dato perdido en el sistema, definiendo por "dato perdido" los casos en que hubo cierta omisión de información. Así, los datos presentados corresponden a un total de 210 participantes con edades comprendidas entre los 6 y los 16 años, con un promedio de edad de 9.4 años y una desviación estándar de 1.7.

El tiempo destinado a la observación y análisis de los registros nos permitió estimar las capacidades de los participantes, su desarrollo y su evolución.

\section{Resultados}

Variables sociodemográficas de interés

El $63.8 \%$ fue de sexo masculino y el $36.2 \%$ de sexo femenino, lo cual evidencia la existencia de una disparidad de género a favor de los varones en cuanto a la preferencia de las habilidades deportivas. Por otro lado, la mayoría de los participantes provienen de familias en las que no es habitual la práctica deportiva. Ello se constata al recuperar las cantidades que indican que solo el 27\% de las madres y el $27.6 \%$ de los padres de los participantes han practicado deportes. Es decir, estamos ante una situación de familias sedentarias.

Frecuencia de la práctica deportiva

Los datos muestran un patrón de concentración de los mayores porcentajes entre los tres y seis días para un porciento acumulado de $84.9 \%$ (ver tabla 2).

Tabla 2. Frecuencia semanal de práctica deportiva.

Días dedicados al deporte

\begin{tabular}{cc}
\hline 1 día & $5.9 \%$ \\
\hline 2 días & $4.6 \%$ \\
\hline 3 días & $23.0 \%$ \\
\hline 4 días & $28.3 \%$ \\
\hline 5 días & $17.8 \%$ \\
\hline 6 días & $15.8 \%$ \\
\hline 7 días & $2.6 \%$ \\
\hline
\end{tabular}

Fuente: Base de datos Maracas 2019. 
Otro aspecto asociado es la cantidad de tiempo diario dedicado al deporte, habiéndose identificado un patrón de concentración de los porcentajes entre los 60 y los 120 minutos por cada práctica, con porciento acumulado de $62.5 \%$.

Frecuencia de las inteligencias múltiples y habilidades para la vida

La inteligencia más frecuente en esta muestra fue la kinestésica corporal (22.4\%), sin embargo, también se manifiestan y estimulan las otras siete inteligencias (ver figura 1). Por otro lado, la habilidad más identificada fue la físico-motora $(35.5 \%)$ y, de la misma forma, se detectan las demás habilidades (ver figura 2).

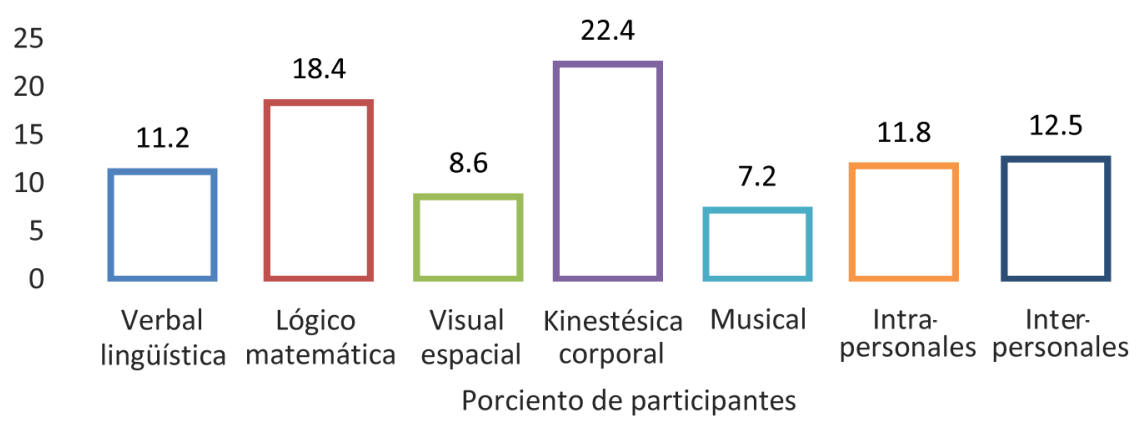

Figura 1. Inteligencias más desarrolladas en los participantes.

Fuente: Base de datos Maracas 2019.

El análisis de las frecuencias de las inteligencias múltiples y habilidades para la vida brinda al profesor(a) un punto de partida sobre los alumnos(as) con quienes trabajará. De esta forma y a manera de diagnóstico, podemos identificar y observar las inteligencias y habilidades con mayor frecuencia. Así mismo se pueden abordar contenidos y estrategias didácticas para favorecer los estilos de aprendizaje desde sus capacidades más desarrolladas, pero también las menos estimuladas o áreas de oportunidad, poniendo especial atención en las formas de abordaje para favorecer los procesos de enseñanza-aprendizaje.

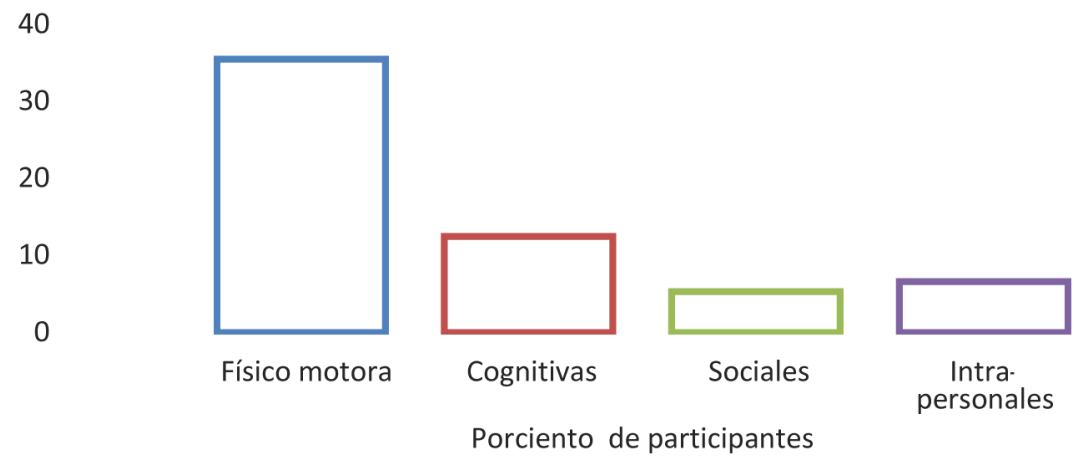

Figura 2. Habilidades más desarrolladas en los participantes.

Fuente: Base de datos Maracas 2019. 


\section{DIsCUSIÓN}

La disparidad de género en la inscripción puede ser entendida por la teoría de la actividad histórico-cultural en tanto los sistemas de comunicación y actividad están culturalmente condicionados, y en este condicionamiento la variable género puede ser esencial, creando orientaciones en los sujetos masculinos que los acercan en mayor proporción a las actividades deportivas que en aquellos de género femenino. Esto implica que las estrategias de comunicación para promocionar la corporeidad mediante la oferta de actividades deben diversificarse con intención desde su planeación y estrategias que permitan incluir de manera más integral a las féminas.

La importancia del componente histórico se refleja en que la mayoría de los participantes provienen de familias en las que, aunque no se practica el deporte, este sí es una actividad ponderada, en tanto se invierte en que los hijos asistan a ligas y clubes deportivos, reflejándose lo anterior, además, en una frecuencia de práctica semanal moderada. Ello entonces crea las condiciones internas de la situación social del desarrollo y fomenta situaciones de actividad y comunicación que potencian el interés hacia la educación física, lo cual allana el camino para estimular las inteligencias múltiples y habilidades para la vida de los participantes desde un escenario de educación no formal, con transferencia al ámbito de la educación formal.

Asimismo, en cuanto a la frecuencia de las habilidades para la vida identificadas (cognitivas, sociales, intrapersonales y físico-motoras), el resultado era esperado cuando se montan escenarios de aprendizaje con eje deportivo, en tanto los sujetos suelen elegir la participación en aquellas actividades para las que se sienten hábiles, y al mismo tiempo se identifican áreas de oportunidad susceptibles de desarrollo. Resultó interesante que al elaborar tablas de contingencia no se encontró asociación de dependencia entre el tiempo semanal dedicado al deporte y la frecuencia con las habilidades e inteligencia identificadas como más frecuentes.

La salud debería tener un tratamiento transversal en el currículo de la educación básica, ya que al ser multifactorial se requiere del apoyo de múltiples aspectos, uno de ellos es el deporte como mediación pedagógica a fin de coadyuvar y fortalecer los vacíos de la educación física en el sistema escolarizado, cuyo tratamiento desde hace varias décadas ha sido limitado en cuanto a tiempo (entre una y dos veces por semana) y forma (escaso énfasis a la corporeidad, la salud y habilidades para la vida); los indicadores de obesidad y violencia dan cuenta de ello. Es decir, la salud tiene que ser abordada desde todas las vertientes escolares del educando, a fin de incorporar de manera activa los contenidos conceptuales, procedimentales y actitudinales a lo largo de la educación básica. Es urgente adoptar mejores prácticas que puedan favorecer la educación física con el objeto de que los alumnos logren comprender e incorporar verdaderamente estilos de vida saludables y permita disminuir paulatinamente los elevados indicadores de obesidad y violencia que aquejan a la sociedad mexicana y en particular a la juventud juarense. 


\section{CONCLUSIÓN}

El presente trabajo de investigación es solo un reflejo de la importancia y eficacia de los campamentos de verano educativos con enfoque multidisciplinar, ya que su planeación, su forma interactiva, espontánea, motivadora y al aire libre suele ser un escenario de aprendizaje social para los niños y jóvenes. Históricamente, se ha observado que el deporte con fines educativos promueve aprendizajes no solo para la vida social, sino también favorece habilidades para la vida (desarrollo cognitivo, intrapersonal, social y motor), fortaleciendo con ello la formación integral, uno de los principales objetivos de la educación básica en México.

Se sugiere contemplar actividades similares a las del campamento de verano sociocultural MARACAs en las clases de Educación Física, como un complemento que favorezca la paridad de género entre los participantes, propiciando ambientes con actividades mixtas como gimnasia aeróbica, danza aérea, porra, baile moderno, activaciones físicas, entre otras.

Asimismo, incorporar la educación física como un espacio de aprendizaje permite identificar y potenciar, por un lado, las inteligencias múltiples de los niños y jóvenes de una manera divertida, desde lo multidisciplinar, y por otro, las habilidades para la vida (cognitivas, sociales, personales, físico-motrices) con enfoque integrador en el cual el participante experimenta diferentes formas de aprender y solucionar problemáticas a partir del juego. Es decir, el juego se convierte en un elemento mediador del aprendizaje (Juárez, 2012).

También, así como el programa MARACAS ofrece la posibilidad de observar cómo una acción con resultados positivos para la prevención de la violencia y la delincuencia entre pares, favorece la reconstrucción del tejido social y la construcción y desarrollo de la comunidad (Cruz, 2014). En este sentido, queda de manifiesto que la educación física, las actividades después de escuela o campamentos de verano educativos, resultan indispensables para la formación integral de los educandos y, sobre todo, resultan ser escenarios ideales para incluir la corporeidad como una estrategia de preservación de la salud en escolares en contextos educativos tanto formales como no-formales.

Finalmente, será importante continuar investigando nuevas formas metodológicas y diseños de estudio con enfoque crítico, los impactos reales que tienen los estímulos que usan al deporte y la educación física como medio y elemento formador en infantes y adolescentes, a fin de seguir encontrando evidencia que potencie el desarrollo integral de los niños y jóvenes en edad escolar.

En definitiva, es recomendable incorporar un mayor énfasis en la corporeidad como parte imprescindible en el desarrollo integral, a fin de estimular, desarrollar y potenciar las habilidades y talentos de quienes lo practican. De esta forma será posible empezar a incidir en los graves indicadores de sobrepeso, obesidad y violencia en niños y jóvenes en el estado de Chihuahua, particularmente en Ciudad Juárez. Los altos indicadores mostrados anteriormente, evidencian que las clases de Educación Física 
en el sistema escolarizado de la Secretaría de Educación Pública, en educación básica, en la actualidad se vuelven insuficientes tanto en su práctica como en su impacto.

\section{REFERENCIAS}

Álvarez, J. (2007). Cómo hacer investigación cualitativa: fundamentos y metodología. México: Paidós. Arias, I. (1994). Tres elementos clave en la obra de Vygotski: situación social del desarrollo, zona de desarrollo próximo e interacción entre enseñanza y desarrollo. Su especificidad en la edad escolar vista a través de un estudio de caso y de un estudio grupal. Revista Educacióny Ciencia, 3(10), 17-23. Recuperado de: http://educacionyciencia.org/index.php/ educacionyciencia/article/view/83/pdf.

Breton, D. (2007). El sabor del mundo. Una antropología de los sentidos. Nueva Visión.

Cruz Sierra, S. (2014). Violencia y jóvenes: pandilla e identidad masculina en Ciudad Juárez. Revista Mexicana de Sociología, 76(4), 613-637. Recuperado de: http://www.scielo.org.mx/ $\mathrm{pdf} / \mathrm{rms} / \mathrm{v} 76 \mathrm{n} 4 / \mathrm{v} 76 \mathrm{n} 4 \mathrm{a} 4 . \mathrm{pdf}$.

Ensanut [Encuesta Nacional de Salud y Nutrición] (2018). Encuesta Nacional de Saludy Nutrición 2018. Presentación de resultados. Recuperado de: https://ensanut.insp.mx/encuestas/ ensanut2018/doctos/informes/ensanut_2018_presentacion_resultados.pdf.

Gardner, H. (1987). Estructuras de la mente. La teoría de las inteligencias múltiples. México: Fondo de Cultura Económica.

INEGI [Instituto Nacional de Estadística y Geografía] (2020). Características de las defunciones registradas en México durante enero-agosto 2020. Recuperado de: https://www.inegi.org. $\mathrm{mx} /$ contenidos/saladeprensa/boletines/2021/EstSociodemo/DefuncionesRegistradas2020_Pnles.pdf.

Juárez, R. (2012). El proyecto MARACAs: una estrategia de educación integral para niños y jóvenes que transforma la realidad social a través de la actividad física [Tesis doctoral]. Facultad de Ciencias de la Educación, Universidad de Lleida, España.

McKernan, J. (2000). Investigación-acción y currículum. Madrid: Morata.

SEP [Secretaría de Educación Pública] (2017). Aprendizajes clave para la educación integral: plan y programas de estudio, orientaciones didácticas y sugerencias de evaluación. Ciudad de México: SEP.

SEP (2019). Sistema Nacional de Información Estadística Educativa. Recuperado de: http:/ / www. planeacion.sep.gob.mx/principalescifras/.

UNESCO [Organización de las Naciones Unidas para la Educación, la Ciencia y la Cultura] (2018). Hacia una estrategia nacional para la prestación de educación física de calidad en el nivel básico del sistema educativo mexicano. Recuperado de: https://unesdoc.unesco.org/ark:/48223/pf00 00264037? posInSet=1\&queryId=N-EXPLORE-97a3e811-5aac-47f2-8f72-4c259f8aa7fe.

Cómo citar este artículo:

Juárez Lozano, R., Lara Rodríguez, L. M., y Medrano Donlucas, G. (2020). La corporeidad como estrategia para el desarrollo de habilidades y preservación de la salud en escolares en la nueva normalidad. RECIE. Revista Electrónica Científica de Investigación Educativa, 5(1), pp. 227-236. doi: doi.org/10.33010/recie.v5i1.1053. 